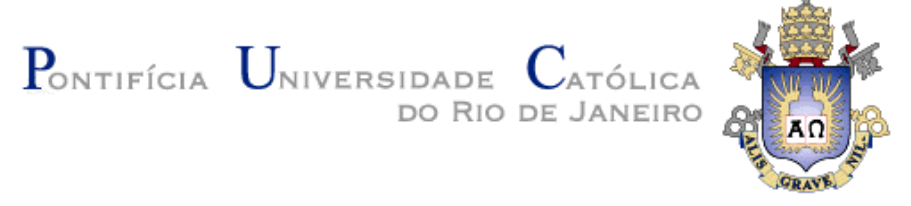

Walace Ferreira de Arantes

\begin{abstract}
Avaliação metrológica da comparação interlaboratorial da calibração de medidores ultra-sônicos.
\end{abstract}

Dissertação de Mestrado

Dissertação apresentada como requisito parcial para obtenção do título de Mestre pelo Programa de PósGraduação em Metrologia para Qualidade e Inovação / PUC-Rio.

Orientadores:

Prof. Alcir de Faro Orlando

Departamento de Engenharia Mecânica / PUC-Rio Programa de Pós-graduação em Metrologia / PUC-Rio

Eng. Valter Yoshihiko Aibe

INMETRO (Instituto Nacional de Metrologia, Normalização e Qualidade Industrial)

Rio de Janeiro, outubro de 2006 
Walace Ferreira de Arantes

\title{
Avaliação metrológica da comparação interlaboratorial da calibração de medidores ultra-sônicos.
}

\begin{abstract}
Dissertação apresentada como requisito parcial para obtenção do grau de Mestre pelo Programa de Pósgraduação em Metrologia do Centro Técnico Científico da PUC-Rio. Aprovada pela Comissão Examinadora e homologada pela Coordenação Setorial de PósGraduação, formalizado pelas respectivas assinaturas.
\end{abstract}

Comissão Examinadora:

Prof. Alcir de Faro Orlando Orientador Departamento de Engenharia Mecânica / PUC-Rio Programa de Pós-graduação em Metrologia / PUC-Rio Pontifícia Universidade Católica do Rio de Janeiro

Eng. Valter Yoshihiko Aibe Co-orientador INMETRO (Instituto Nacional de Metrologia e Qualidade Industrial)

Prof. Maurício Nogueira Frota Programa de Pós-graduação em Metrologia / PUC-Rio

Pontifícia Universidade Católica do Rio de Janeiro

Eng. Sidney Stuckenbruck Olympus Software Científico e Engenharia Ltda.

Prof. Washington Braga Filho Departamento de Engenharia Mecânica (DEM / PUC-Rio Pontifícia Universidade Católica do Rio de Janeiro

Coordenação Setorial de Pós-Graduação:

Prof. José Eugênio Leal Coordenador Setorial de Pós-Graduação do Centro Técnico Científico (PUC-Rio)

Rio de Janeiro, 17 de outubro de 2006. 
Todos os direitos reservados. É proibida a reprodução total ou parcial do trabalho sem autorização da universidade, do autor e do orientador.

\section{Walace Ferreira de Arantes}

Graduado em Engenharia Mecânica pela F.T.E. Souza Marques, em 1994. Engenheiro atuando na Gestão da Qualidade, de 1994 a 1997 da empresa Termolite Ltda. Gerente da Qualidade de 1999 a 2000 na empresa Valeq Válvulas e Equipamentos Ind. Ltda., e como Engenheiro de Equipamentos, a partir de 2001, na Unidade de Negócios da Bacia de Campos / Petrobras

Ficha Catalográfica

Arantes, Walace Ferreira de

Avaliação metrológica da comparação interlaboratorial da calibração de medidores ultra-sônicos / Walace Ferreira de Arantes ; orientadores: Alcir de Faro Orlando, Valter Yoshihiko Aibe. - 2007.

142 f. : il. ; $30 \mathrm{~cm}$

Dissertação (Mestrado em Metrologia para Qualidade e Inovação) - Pontifícia Universidade Católica do Rio de Janeiro, Rio de Janeiro, 2007.

Inclui bibliografia

1. Metrologia - Teses. 2. Medição. 3. Tempo de trânsito. 4. Sistema de medição. 5. Sistema de calibração. 6. Petróleo. I. Orlando, Alcir de Faro. II. Aibe, Valter Yoshihiko. III. Pontifícia Universidade Católica do Rio de Janeiro. Programa de Pós-Graduação em Metrologia para Qualidade Industrial. VI. Título. 
Dedico este trabalho a minha esposa Dolores pela compreensão e paciência durante todo período do curso e a minha filhinha Vitória, que é um verdadeiro presente de Deus. 


\section{Agradecimentos}

A Deus pela realização desta obra e de um sonho.

Aos meus pais que sempre me deram o apoio necessário para eu estudar.

Aos meus amigos Marcelo Almeida e Sebastião da Rosa pelas orações intercessórias.

Ao Grupo de Medição da UN-BC e demais colegas da Petrobras que prestaram uma grande contribuição.

À Petrobras pelo incentivo ao desenvolvimento profissional e às empresas participantes, que cederam suas instalações para realização dos trabalhos experimentais.

Ao professor Alcir, por acreditar na proposta e ser um grande incentivador.

Ao Engenheiro Valter Aibe, do INMETRO, por participar na fase experimental e final da pesquisa.

Ao professor Maurício Frota, que sempre esteve atento às demandas do curso.

Aos mestrandos de Metrologia.

E por fim, aos componentes da banca que deram suas contribuições para melhoria deste trabalho e pela participação dos mesmos nesse momento tão importante da minha vida. 


\section{Resumo}

Arantes, Walace Ferreira; Orlando, Alcir de Faro. Avaliação metrológica da comparação interlaboratorial da calibração de medidores ultrasônicos. Rio de Janeiro, 2006. 142p. Dissertação de Mestrado - Programa de Pós-Graduação em Metrologia, Pontifícia Universidade Católica do Rio de Janeiro.

O presente trabalho refere-se à avaliação metrológica de um mesmo medidor ultra-sônico de vazão de líquido pelo método de tempo de trânsito, quando instalado em dois sistemas distintos de calibração, pertencentes, respectivamente, a dois laboratórios acreditados, ambos operando em conformidade com as especificações técnicas de instalação do fabricante e segundo os requisitos mínimos especificados em normas e recomendações internacionais. A pesquisa investigou o efeito na calibração introduzido por uma válvula e uma curva longa de $90^{\circ}$ instaladas imediatamente à montante do trecho reto de medição, que tem o seu comprimento atendendo as condições mínimas especificadas nas normas aplicáveis. Concluiu-se pela necessidade de se utilizar pelo menos 40 diâmetros nominais $(40 \mathrm{DN})$ de comprimento de trecho reto, para que a influência da presença da válvula e da curva longa possa ser considerada desprezível. O desenvolvimento do presente trabalho foi motivado pela crescente aplicação de medidores ultra-sônicos de vazão na indústria de petróleo e pela urgente necessidade de se contribuir para o avanço do conhecimento desta tecnologia e da normalização aplicável. Nesse sentido, o presente trabalho contribui:

(i) para o melhor entendimento da tecnologia de medição e do processo de calibração de medidores ultra-sônicos de vazão de líquidos;

(ii) para o avanço da normalização no setor, uma vez que inclui uma análise crítica de requisitos mínimos normalizados;

(iii) para aprimoramento dos critérios de acreditação de laboratórios que realizam a calibração de medidores de vazão, já que avaliou desvios de calibração ocorridos em distintas instalações em conformidade com as normas aplicáveis.

\section{Palavras-chave}

Metrologia; medição; tempo de trânsito; sistema de medição; sistema de calibração; petróleo. 


\section{Abstract}

Arantes, Walace Ferreira; Orlando, Alcir de Faro. Metrological evaluation of the interlaboratorial comparison of the ultrasonic flow meter calibration. Rio de Janeiro, 2006. 142p. M.Sc. Dissertation - Graduate Metrology. MQI. Pontifical Catholic University of Rio de Janeiro, Brazil.

This work deals with the metrological evaluation of the same ultrasonic flow meter by the transit time method, when installed in two different calibrating systems, belonging, respectively, to two accredited laboratories, both operating according to the manufacture's installation technical specification, and following the minimum requirements, as specified in international standards and recommendations. The research investigated the effect in calibration due to the installation of a valve and a long $90^{\circ}$ curve, immediately upstream of the measurement test section, with its length dimensioned according to the minimum requirements of the applicable standards. It was concluded that a longer straight sections (at least 40 diameters) should be specified, so that the influence of the installation of the valve and the curve could be neglected. The development of the present work was motivated by the growing utilization of ultrasonic flow meters by the oil industry, and by the urgent need to contribute to the advance of the technology and the applicable standardization. In this aspect, this work contributes to:

(i) a better understanding of the measurement technology and of the procedure of ultrasonic liquids flow meters;

(ii) the advance of the technology standardization, since it includes a critical analysis of the minimum requirements, as specified by the standards;

(iii) improving the laboratory accreditation criteria for flow meter calibration, because the influence of the installation in the calibration was experimentally shown.

\section{Key-words}

Metrology; measurement; time of transit; measurement system; calibration system; petroleum. 


\section{Sumário}

1 Introdução 19

1.1 O papel da Metrologia como estratégia de competitividade 20

1.2 Legislação aplicável à medição de petróleo 21

1.2.1 Portaria Conjunta No 1, de 19 de Junho de 2000. (ANP / INMETRO) 22

1.2.2 Portaria INMETRO N064, de 11 de abril de 2003

1.2.3 Portaria INMETRO N²34, de 12 de Agosto de 2003

1.2.4 Lei No 9.847, de 26 de Outubro de 1999

1.2.5 Lei No 9.478, de 6 de Agosto de 1997

1.3 Recomendação internacional aplicável à medição de petróleo 24

1.4 Impactos decorrentes da legislação de petróleo 24

1.5 O estado da arte 25

1.6 Motivação para desenvolvimento do presente trabalho 34

1.7 Objetivos 36

1.8 Estruturação 37

2 Fundamentos teóricos

38

2.1 Princípio geral de medição de vazão por ultra-som 38

2.1.1 Tipos de medição de vazão por ultra-som 38

2.1.1.1 Medição por ultra-som — efeito Doppler 39

2.1.1.2 Medição por ultra-som — correlação cruzada 40

2.1.1.3 Medição por ultra-som — tempo de trânsito 41

2.1.2 Geração dos sinais ultra-sônicos 42

2.1.3 Concepções e arranjos do medidor 43

2.1.4 Arranjo de transdutor ultra-sônico 44

2.1.4.1 Arranjo de trajetória única $\quad 45$

2.1.4.2 Cálculo da vazão usando trajetória simples 46

2.1.4.3 Arranjo de trajetória múltipla $\quad 47$

2.1.4.4 Cálculo da vazão usando trajetórias múltiplas 48 
2.2 Medição ultra-sônica de vazão pelo método de tempo de trânsito 48

2.2.1 Princípio de medição ultra-sônica por tempo de trânsito 49

2.2.1.1 Medidor de vazão ultra-sônico — definição 51

2.2.1.2 Totalizador de vazão — definição 51

2.2.2 Método da diferença de tempo de trânsito 51

2.2.2.1 Método do tempo de trânsito direto 52

2.2.2.2 Método da Freqüência de repetição do pulso ("Sing-around") 53

2.2.2.3 Métodos de Deslocamento de Fase 53

2.2.2.3.1 Método "Diferença de Fase" 53

2.2.2.3.2 Método "Controle de Fase" 54

2.2.3 Cálculo da vazão volumétrica $\left(q_{v}\right)$ utilizando arranjo de

trajetórias diametrais $\quad 55$

2.2.4 Cálculo da vazão volumétrica (qv) utilizando arranjo multitrajetórias em planos paralelos 56

2.3 Fatores que influenciam na exatidão e na repetitividade de medidores ultra-sônicos pelo método de tempo de trânsito 57

2.4 Requisitos de instalação 58

2.5 Sistemas de calibração 59

2.5.1 Sistema Tanque provador 59

2.5.2 Sistema com medidor padrão (master meter) 60

2.6 Considerações normativas sobre os sistemas de calibração 61

2.6.1 API MPMS 4.8 - System Prover-Operation of Proving Systems 61

2.6.2 API MPMS 4.5 - System Prover - Master Meter Provers. 62

2.6.3 API MPMS 5.8 - Measurement of Liquid Hydrocarbons by Ultrasonic Flowmeters Using Transit Time Technology 63

3 Desenvolvimento Experimental 64

3.1 Metodologia da pesquisa 64

$\begin{array}{lll}3.2 & \text { Sistemas de calibração } & 65\end{array}$

$\begin{array}{lll}3.3 & \text { Características do medidor calibrado } & 67\end{array}$

3.4 Métodos de calibração e de determinação dos resultados 67

3.4.1 Procedimento Experimental utilizado pelo Laboratório A 68

3.4.1.1 Descrição dos Métodos realizados pelo Laboratório A 68

3.4.1.2 Objetivos dos ensaios realizados pelo Laboratório A 70 
3.4.1.3 Faixa de vazão calibrada nos Métodos realizados pelo Laboratório A 71

3.4.2 Procedimento Experimental utilizado pelo Laboratório B 71

3.4.3 Descrição dos Métodos realizados pelo Laboratório B 71

4 Análise de resultados

74

4.1 Resultados obtidos no Laboratório A

4.2 Resultados obtidos no Laboratório B 82

4.3 Análise global dos resultados 90

4.3.1 Influência de instalações 90

4.3.1.1 Analise dos resultados do Laboratório A, Métodos-1 e 2. 91

4.3.1.2 Análise dos resultados do Laboratório B, Método-3 92

4.3.1.3 Análise dos resultados - Lab. A e Métodos-1 e 2 x Lab. B Método-3 92

4.3.2 Influência do procedimento de calibração 93

4.3.3 Influência do volume provado 94

4.3.3.1 Análise dos resultados do Lab. A Métodos-1 e 2.

4.3.3.2 Análise dos resultados - Lab. A Método-2 $\times$ Lab. B Método-3 95

4.3.4 Influência do atraso na geração de sinal (delay) 95

4.3.4.1 Observações relevantes sobre as Fig. 45, 48 e $51 \quad 96$

4.3.4.2 Observações relevantes sobre as Fig. 46, 49 e 52

4.3.4.3 Observações relevantes sobre as Fig. 47, 50 e $53 \quad 97$

4.3.5 Outras observações relevantes 97

5 Conclusões e recomendações

98

5.1 Conclusões 98

5.2 Recomendações 99

5.3 Sugestões para futuros trabalhos 100

6 Referências bibliográficas 101

Apêndice - Fundamentos da Medição de Vazão 102

Fundamentos da medição de vazão 102

$\begin{array}{ll}\text { Regime de escoamento } & 104\end{array}$

Velocidade média - conceito 105 
$\begin{array}{ll}\text { Sistemas de medição de vazão } & 108\end{array}$

Medidores para a indústria do petróleo 110

Medidor de deslocamento positivo (DP) 110

Medidor do tipo disco de nutação 111

Medidor do tipo paleta rotativa e deslizante 111

Medidores de lóbulos rotativos e engrenagens 113

Medidor ultra-sônico 113

Cenário mundial da medição de vazão por ultra-som 114

Anexo 1 - Dados da calibração no Laboratório B, pelo Método-4 período 0 a $177 \mathrm{~s}$

Anexo 2 - Dados da calibração no Laboratório B, pelo Método-4 período 24,5 a $162,5 \mathrm{~s}$.

Anexo 3 - Dados da calibração no Laboratório B, pelo Método-4 período 0 a $102 \mathrm{~s}$

Anexo 4 - Dados da calibração no Laboratório B, pelo Método-4 período 33,5 a $89 \mathrm{~s}$

Anexo 5 - Dados da calibração no Laboratório B, pelo Método-4 período 0 a $84 \mathrm{~s}$

Anexo 6 - Dados da calibração no Laboratório B, pelo Método-4 período 22,5 a $58,5 \mathrm{~s}$ 


\section{Lista de tabelas}

Tabela 1 - Estimativa do desvio padrão para uma série de calibração

Tabela 2 - Objetivos dos testes realizados pelo Método-1 e pelo Método-2 no Laboratório A

Tabela 3 - Condições dos ensaios no Laboratório A

Tabela 4 - Erros relativos (\%) dos ensaios no Laboratório A. 75

Tabela 5 - Repetitividade (\%) dos ensaios no Laboratório A. 75

Tabela 6 - Erro x Vazão Nominal obtidos no Lab. B, Método-3 82

Tabela 7 - Repetitividade x Vazão Nominal obtidos no Lab. B, Método-3 82

Tabela 8 - Erros total e parcial na vazão nominal de $300 \mathrm{~m}^{3} / \mathrm{h} \quad 86$

Tabela 9 — Erros total e parcial na vazão nominal de $400 \mathrm{~m}^{3} / \mathrm{h} \quad 88$

Tabela 10 — Erros total e parcial na vazão nominal de $500 \mathrm{~m}^{3} / \mathrm{h} \quad 90$

Tabela 11 - Dados comparativos dos principais medidores de vazão

— caracterização do fluido

103

Tabela 12 - Dados comparativos dos principais medidores de vazão — especificações e requisitos de instalação

Tabela 13 - Dados comparativos dos principais medidores de vazão - economicidade, vantagens e desvantagens.

Tabela 14 - Dados do período de 0 a 177 segundos na $2^{\text {a }}$ corrida de $300 \mathrm{~m}^{3} / \mathrm{h}(13 / 07 / 06)$

Tabela 15 - Dados da calibração no Laboratório B, pelo Método-4, do período de 24,5 a 162,5 segundos na $2^{\text {a }}$ corrida de $300 \mathrm{~m}^{3} / \mathrm{h}(13 / 07 / 06)$

Tabela 16 - Dados da calibração no Laboratório B, pelo Método-4, do período de 0 a 102,0 segundos na $1^{\text {a }}$ corrida de $400 \mathrm{~m}^{3} / \mathrm{h}$.

Tabela 17 - Dados da calibração no Laboratório B, pelo Método-4, do período de 33,5 a 89,5 segundos na $1^{\text {a }}$ corrida de $400 \mathrm{~m}^{3} / \mathrm{h}$

Tabela 18 - Dados da calibração no Laboratório B, pelo Método-4, do período de 0 a 84,0 segundos na $1^{\text {a }}$ corrida com vazão nominal de $500 \mathrm{~m}^{3} / \mathrm{h}$

Tabela 19 - Dados da calibração no Laboratório B, pelo Método-4, no período de 22,5 a 58,5 segundos na $1^{\text {a }}$ corrida com vazão nominal de $500 \mathrm{~m}^{3} / \mathrm{h} 141$ 


\section{Lista de Figuras}

Figura 1- Estimativa do impacto econômico devido a erros na medição de petróleo

Figura 2 - Mercado mundial de medidores utilizados para medição de líquido e gás (Fonte:Emerson Process).

Figura $3 \mathrm{a}$ - Trecho reto à montante com 20 diâmetros e com retificador de fluxo

Figura $3 b$ - Trecho reto à montante com 20 diâmetros e sem retificador de fluxo

Figura $3 \mathrm{c}$ - Trecho reto à montante com 10 diâmetros e com retificador de fluxo

Figura $3 \mathrm{~d}$ - Trecho reto à montante com 10 diâmetros e sem retificador de fluxo

Figura $3 \mathrm{e}$ - Configuração típica de um sistema de medição fiscal (switchback)

Figura 4 - teste básico no medidor ultra-sônico

Figura 5 - Efeito de instalação com redução no medidor e com retificador de fluxo

Figura 6 - Efeito de instalação com redução no medidor e sem retificador de fluxo

Figura 7 - Efeito de instalação com acidentes a montador do medidor e com retificador de fluxo

Figura 8 - Efeito de instalação com acidente de montador do medidor e sem retificador de fluxo

Figura 9 - Volume provado $\mathrm{x}$ tamanho do medidor

Figura 10 - Perfil de fluxo e desvio padrão da velocidade de cada trajetória 33

Figura 11 - Perfil de fluxo após a remoção da obstrução

Figura 12 - Efeitos da partida (início) de calibração - antes e depois do provador instalado à montante do trecho reto de medição.

Figura 13 - Diferenças de erros relativos entre laboratórios

Figura 14 - Princípio de Funcionamento dos Medidores Tipo Doppler 
Figura 15 - Diagrama esquemático de um medidor ultra-sônico por correlação cruzada

Figura 16 - Princípio de funcionamento dos medidores tipo tempo de trânsito 41

Figura 17 - Configuração de um transdutor piezoelétrico.

Figura 18 - Padrão do feixe medido de um transdutor com diâmetro de saída de $2 \mathrm{~cm}$ em uma frequiência de operação de $162 \mathrm{kHz}$. 43

Figura 19a - Transdutor em contato com o fluido (retraído) 44

Figura 19b - transdutor em contato com o fluido (faceado) 44

Figura 19c - transdutor montado do lado externo do tubo (arranjo clamp-on) 44

Figura 19d - transdutor intrusivo no fluxo 45

Figura 20a - Transmissão direta 45

Figura 20b - Transmissão indireta (refletida na parede do tubo: trajetória em "V") 45

Figura 20c - Transmissão indireta (refletida na parede do tubo: trajetória em “W”) 46

Figura 20d — Diâmetro inclinado $\quad 46$

Figura 20e - Corda inclinada $\quad 46$

Figura 20f — Transdutores montados axialmente $\quad 46$

$\begin{array}{ll}\text { Figura 21a - Plano único } & 47\end{array}$

Figura 21b - Cruzado simétrico $\quad 47$

Figura 21c - Cruzado assimétrico $\quad 48$

Figura 21d - Par casado $\quad 48$

Figura 22 - Medição ultra-sônica pelo método de tempo de trânsito 49

Figura 23 - Configuração do tipo feixes paralelos 50

Figura 24 - Configuração matricial de trajetórias acústicas 51

Figura 25 - Fases dos sinais ultra-sônicos a montante e a jusante $\quad 54$

Figura 26 - Valor aproximado para kh em função de $\operatorname{Re}_{\mathrm{D}}$

Figura 27 - Tanque provador do tipo aberto 60

Figura 28 - Sistema típico com medidor padrão (master meter). 62

Figura 29 - Sistema de calibração do Laboratório A 66

Figura 30 - Sistema de calibração do Laboratório B 66

Figura 31 - Erro x vazão nominal no Ensaio C1T1 76

Figura 32 - Repetitividade x vazão nominal no ensaio C1T1 76 
Figura 33 - Erro x vazão nominal no ensaio C2T1 77

Figura 34 - Repetitividade x vazão nominal no ensaio C2T1 77

Figura 35 - Erro x vazão nominal no ensaio C2T2 78

Figura 36 - Repetitividade x vazão nominal no ensaio C2T2 78

Figura 37 — Erro x Vazão nominal no ensaio C2T3 79

Figura 38 - Repetitividade x Vazão nominal no ensaio C2T3 79

Figura 39 - Erro (\%) x vazão nominal no ensaio C2T4 80

Figura 40 - Repetitividade (\%) x vazão nominal no ensaio C2T4 80

Figura 41 - Perfil de escoamento no início de um ensaio partindo do fluido $\begin{array}{ll}\text { em repouso } & 81\end{array}$

Figura 42 - Perfil de escoamento durante a corrida com vazão estabilizada. $\quad 82$

Figura 43 - Resultados de Erro x Vazão Nominal obtidos no Laboratório B

, Método-3 83

Figura 44 - Repetitividade x vazão nominal no Laboratório B, Método-3 83 Figura 45 - Influência do atraso na geração de pulsos do medidor ultra-sônico, na vazão nominal de $300 \mathrm{~m}^{3} / \mathrm{h}$. $\quad 85$

Figura 46 - Influência da escolha do período de contagem de pulsos do medidor ultra-sônico no erro de medição, na vazão nominal de $300 \mathrm{~m}^{3} / \mathrm{h}$.

Figura 47 - Influência no erro de medição com delay e sem delay, na vazão nominal de $300 \mathrm{~m}^{3} / \mathrm{h}$.

Figura 48 - Influência do atraso na geração de pulsos do medidor

ultra-sônico no erro de medição, na vazão nominal de $400 \mathrm{~m}^{3} / \mathrm{h}$.

Figura 49 - Influência da escolha do período de contagem de pulsos do medidor ultra-sônico no erro de medição, na vazão nominal de $400 \mathrm{~m}^{3} / \mathrm{h}$

Figura 50 - Influência no erro de medição, com delay e sem delay, na vazão nominal de $400 \mathrm{~m}^{3} / \mathrm{h}$

Figura 51 - Influência do atraso na geração de pulsos do medidor

ultra-sônico no erro de medição, na vazão nominal de $500 \mathrm{~m}^{3} / \mathrm{h}$.

Figura 52 - Influência da escolha do período de contagem de pulsos do medidor ultra-sônico no erro de medição, na vazão nominal de $500 \mathrm{~m}^{3} / \mathrm{h}$

Figura 53 - Influência no erro de medição, com delay e sem delay, na vazão nominal de $500 \mathrm{~m}^{3} / \mathrm{h}$.

Figura 54 - Perfil de escoamento em regimes laminar e turbulento 
Figura 55 - Relação Vm / Vc em função do número de Reynolds

Figura 56 - Arquitetura típica de um sistema de medição de petróleo em linha

Figura 57 - Medidor do tipo disco de nutação

Figura 58 - Medidor de palhetas rotativas deslizante

Figura 59 - Medidor de palhetas rotativas com volume constante 


\section{Lista de símbolos}

\begin{tabular}{|c|c|c|}
\hline Símbolos & Descrição & Unidade SI \\
\hline A & Área da seção transversal & $\mathrm{m}^{2}$ \\
\hline $\mathrm{c}$ & Velocidade de propagação do fluido escoando & $\mathrm{m} / \mathrm{s}$ \\
\hline $\mathrm{c}_{\mathrm{o}}$ & Velocidade do som no fluido em repouso & $\mathrm{m} / \mathrm{s}$ \\
\hline $\mathrm{D}$ & Diâmetro interno da tubulação & $\mathrm{m}$ \\
\hline $\mathrm{d}$ & Distância de influência & $\mathrm{m}$ \\
\hline $\mathrm{f}$ & Freqüência & $s^{-1}$ \\
\hline $\mathrm{f}_{\mathrm{r}}$ & Freqüência Doppler defasada & $\mathrm{Hz}$ \\
\hline$f_{t}$ & Freqüência transmitida & $\mathrm{Hz}$ \\
\hline $\mathrm{i}$ & Inteiro & adimensional \\
\hline $\mathrm{K}_{\mathrm{c}}$ & Coeficiente adimensional ou $\mathrm{n}^{\circ}$ crítico de Reynolds & adimensional \\
\hline $\mathrm{k}_{\mathrm{h}}$ & Fator de correção da distribuição de velocidade & adimensional \\
\hline $\mathrm{L}$ & Comprimento de influência & $\mathrm{m}$ \\
\hline $\mathrm{L}_{\mathrm{p}}$ & Comprimento da trajetória & $\mathrm{m}$ \\
\hline $\mathrm{m}$ & Inteiro & adimensional \\
\hline $\mathrm{n}$ & Inteiros $(1,2,3, \ldots)$ & adimensional \\
\hline $\mathrm{q}_{\mathrm{v}}$ & Vazão volumétrica & $\mathrm{m}^{3} / \mathrm{s}$ \\
\hline $\operatorname{Re}_{\mathrm{D}}$ & Número de Reynolds (relacionado a D) & adimensional \\
\hline $\mathrm{t}$ & Tempo de trânsito & $\mathrm{s}$ \\
\hline $\mathrm{v}$ & Velocidade da descontinuidade em relação ao eixo & $\mathrm{m} / \mathrm{s}$ \\
\hline $\mathrm{V}$ & Velocidade local do fluido & $\mathrm{m} / \mathrm{s}$ \\
\hline $\mathrm{V}_{\mathrm{cr}}$ & Velocidade crítica & $\mathrm{m} / \mathrm{s}$ \\
\hline$\overline{\mathrm{V}}$ & $\begin{array}{l}\text { Velocidade média do fluido ao longo da trajetória } \\
\text { acústica }\end{array}$ & $\mathrm{m} / \mathrm{s}$ \\
\hline$\overline{\mathrm{V}}_{\mathrm{a}}$ & Velocidade média axial do fluido & $\mathrm{m} / \mathrm{s}$ \\
\hline $\mathrm{W}_{\mathrm{i}}$ & Peso de medição & \\
\hline $\begin{array}{l}\text { Símbolos } \\
\text { gregos }\end{array}$ & Descrição & Unidade SI \\
\hline$\gamma$ & Ângulo de fase & $\mathrm{rad}$ \\
\hline$\phi$ & Ângulo de inclinação & rad \\
\hline$\theta_{r}$ & Ângulo de recepção & $\mathrm{rad}$ \\
\hline$\theta_{t}$ & Ângulo de transmissão & rad \\
\hline$\lambda$ & Comprimento de onda de uma oscilação ultra-sônica & $\mathrm{m}$ \\
\hline$\Delta t$ & Diferença de tempo de trânsito & $\mathrm{s}$ \\
\hline$\omega$ & Freqüência Cíclica & $\operatorname{rad~s}^{-1}$ \\
\hline$\delta_{f}$ & Intensidade de ultra-som & $\mathrm{Hz}$ \\
\hline$\rho$ & Massa específica do fluido & $\mathrm{kg} / \mathrm{m}^{3}$ \\
\hline$\tau$ & Tempo de integração & $\mathrm{s}$ \\
\hline$v$ & Viscosidade cinemática & $\mathrm{m}^{2} / \mathrm{s}$ \\
\hline
\end{tabular}


AGA

ANP

API

API MPMS

CB-04

EMED

INMETRO

MUS

NEL

$\mathrm{NMi}$

OIML

PETROBRAS

PUC-Rio

RBC

RTM

SI

SPSE

UN-BC

UN-Rio

VIM
American Gas Association (Washingtom, DC, EUA)

Agência Nacional do Petróleo, Gás Natural e Biocombustíveis (Brasil)

American Petroleum Institute, (Washingtom, DC, EUA)

Manual Petroleum Mesurement System

Comitê Brasileiro de Máquinas e Equipamentos Mecânicos (Associação Brasileira de Normas Técnicas, Brasil).

Estação de medição composta, em geral, por elementos primários e secundários de medição.

Instituto Nacional de Metrologia, Normalização e Qualidade Industrial (Brasil).

Medidor ultra-sônico

National Engineering Laboratory (Escócia)

Nederlands Meetinstituut (Holanda)

The International Organization of Legal Metrology (França)

Petróleo Brasileiro S/A

Pontifícia Universidade Católica do Rio de Janeiro (Brasil)

Rede Brasileira de Calibração

Regulamento Técnico de Medição de Petróleo e Gás Natural

Sistema Internacional de Unidades

Societe Du Pipeline Sud Europeen (França)

Unidade de Negócios da Bacia de Campos, Petrobras (Brasil)

Unidade de Negócios do Rio de Janeiro, Petrobras (Brasil)

Vocabulário Internacional de Metrologia 